\title{
Minimum Rank of Graphs Powers Family
}

\author{
Alimohammad Nazari ${ }^{1}$, Marzieh Karimi Radpoor ${ }^{2}$ \\ ${ }^{1}$ Department of Mathematics, University of Arak, Arak, Iran \\ ${ }^{2}$ Institute Scientific and Research Bahar, Hamedan, Iran \\ Email: a-nazari@araku.ac.ir, karimiradpoor@yahoo.com
}

Received January 25, 2012; revised February 27, 2012; accepted March 15, 2012

\begin{abstract}
In this paper we study the relationship between minimum rank of graph $G$ and the minimum rank of graph $G^{j}$ for some families of special graph $G$, where $G^{j}$ is the $j$ th power of graph $G$.
\end{abstract}

Keywords: Minimum Rank; Power of Graphs; Zero Forcing Set

\section{Introduction}

A graph is a pair $G=(V, E)$, where $V$ is the set of vertices (usually $\{1, \cdots, n\}$ or a subset thereof) and $E$ is the set of edges (an edge is a two-element subset of vertices); what we call a graph is sometimes called a simple undirected graph. In this paper each graph is finite and has nonempty vertex set. The order of a graph $G$, denoted $|G|$, is the number of vertices of $G$. A path is a graph $P_{n}=\left(\left\{v_{1}, \cdots, v_{n}\right\}, E\right)$ such that

$$
E=\left\{\left\{v_{i}, v_{i+1}\right\}: i=1, \cdots, n-1\right\} .
$$

A cycle is a graph $C_{n}=\left(\left\{v_{1}, \cdots, v_{n}\right\}, E\right)$ such that $E=\left\{\left\{v_{i}, v_{i+1}\right\}: i=1, \cdots, n-1\right\} \cup\left\{\left\{v_{n}, v_{1}\right\}\right\}$. The length of a path or cycle is the number of its edges. A complete graph is a graph $K_{n}=\left(\left\{v_{1}, \cdots, v_{n}\right\}, E\right)$ such that $E=\left\{\left\{v_{i}, v_{j}\right\}: 1 \leq i<j \leq n\right\}$. A graph $(V, E)$ is bipartite if the vertex set $V$ can be partitioned into two nonempty subsets $U$ and $W$, such that every edge of $E$ has one endpoint in $U$ and one in $W$. A complete bipartite graph is a bipartite graph $K_{p, q}=(U \cup W, E)$ such that $|U|=p$, $|V|=q$ and $E=\{\{u, w\}: u \in U, w \in W\}$.

The line graph of a graph $G=(V, E)$, denoted $L(G)$, is the graph having vertex set $E$, with two vertices in $L(G)$ adjacent if and only if the corresponding edges share an endpoint in $G$. Since we require a graph to have a nonempty set of vertices, the line graph $L(G)$ is defined only for a graph $G$ that has at least one edge.

The corona of $G$ with $H$, denoted $G \circ H$, is the graph of order $|G||H|+|G|$ obtained by taking one copy of $G$ and $|G|$ copies of $H$, and joining all the vertices in the $i$ th copy of $H$ to the $i$ th vertex of $G$. See Figures $\mathbf{6 ,} 7$ for a picture of $K_{6} \circ K_{1}$. Note that $G \circ H$ and $H \circ G$ are usually not isomorphic (in fact, if $|G| \neq|H|$, then $|G \circ H| \neq|H \circ G|)$.

Definition 1.1 The $j$ th power of a graph $G$ is a graph with the same set of vertices as $G$ and an edge between two vertices if there is a path of length at most $j$ between them.

Definition 1.2 For such a matrix, the graph of $A$, denoted $G(A)$, is the graph with vertices $\{1, \cdots, n\}$ and edges $\left\{\{i, j\}: a_{i j} \neq 0,1 \leq i<j \leq n\right\}$. Note that the diagonal of $A$ is ignored in determining $G(A)$. The set of symmetric matrices of graph $G$ (over $R$ ) is defined to be

$$
S(G)=\left\{A \in S_{n}(R): G(A)=G\right\}
$$

The minimum rank of a graph $G$ (over $R$ ) is defined to be

$$
m r(G)=\min \{\operatorname{rank}(A): A \in S(G)\}
$$

For $A \in R^{n \times n}$ the corank of $A$ is the nullity of $A$ and the maximum nullity (or maximum corank) of a graph $G$ (over $R$ ) is defined to be

$$
M(G)=\max \{\operatorname{corank}(A): A \in S(G)\}
$$

Clearly

$$
m r(G)+M(G)=|G| .
$$

More generally, the minimum rank of a simple graph $G$ is defined to be the smallest possible rank over all symmetric real matrices whose ijth entry (for $i \leq j$ ) is nonzero whenever $\{i, j\}$ is an edge in $G$ and is zero otherwise [1,2].

The solution to the minimum rank problem is equivalent to the determination of the maximum multiplicity of an eigenvalue among the same family of matrices [3]. 


\section{Zero Forcing Sets and the Graph Parameter $Z(G)$}

Here we introduce the graph parameter $Z(G)$ as the minimum size of a zero forcing set from [1]. The zero forcing number is a useful tool for determining the minimum rank of structured families of graphs and small graphs [4].

Definition 2.1 Color-change rule:

- If $G$ is a graph with each vertex colored either white or black, $u$ is a black vertex of $G$, and exactly one neighbor $v$ of $u$ is white, then change the color of $v$ to black.

- Given a coloring of $G$, the derived coloring is the result of applying the color-change rule until no more changes are possible.

- A zero forcing set for a graph $G$ is a subset of vertices $Z$ such that if initially the vertices in $Z$ are colored black and the remaining vertices are colored white, the derived coloring of $G$ is all black.

- $Z(G)$ is the minimum of $|Z|$ over all zero forcing sets $Z \subseteq Z(G)$.

For example, an endpoint of a path is a zero forcing set for the path. In a cycle, any set of two adjacent vertices is a zero forcing set.

Corollary 2.2 [1,5] Let $G=(V, E)$ be a graph and let $Z \subseteq V$ be a zero forcing set. Then $M(G) \leq|Z|$, and thus $M(G) \leq Z(G)$.

The Colin deVerdiere-type parameter $\xi$ can be useful in computing minimum rank or maximum nullity (over the real numbers). A symmetric real matrix $M$ is said to satisfy the Strong Arnold Hypothesis provided there does not exist a nonzero symmetric matrix $X$ satisfying:

$$
\begin{aligned}
& \text { - } M X=0 . \\
& \text { - } M \circ X=0 . \\
& \text { - } I \circ X=0 .
\end{aligned}
$$

where $\circ$ denotes the Hadamard (entrywise) product and $I$ is the identity matrix. For a graph $G, \xi(G)$ is the maximum nullity among matrices $A \in S(G)$ that satisfy the Strong Arnold Hypothesis.

It follows that $\xi(G) \leq M(G)$.

A contraction of $G$ is obtained by identifying two adjacent vertices of $G$, and suppressing any loops or multiple edges that arise in this process. A minor of $G$ arises by performing a series of deletions of edges, deletions of isolated vertices, and/or contractions of edges. A graph parameter $\zeta$ is minor monotone if for any minor $G^{\prime}$ of $G, \zeta\left(G^{\prime}\right) \leq \zeta(G)$. The parameter $\xi$ was introduced in [6], where it was shown that $\xi$ is minor monotone. It was also established that $\xi\left(K_{n}\right)=n-1$ and $\xi\left(K_{p, q}\right)=p+1$ (under the assumptions that $p \leq q, 3 \leq q$ ) (see $[5,7]$ ).

Corollary 2.3 [2,6] Let $G$ be a graph.
1) If $K_{p}$ is a minor of $G$, then

$$
M(G) \geq \xi\left(K_{p}\right)=p-1 .
$$

2) If $p \leq q, 3 \leq q$ and $K_{p, q}$ is a minor of $G$, then

$$
M(G) \geq \xi\left(K_{p, q}\right)=p+1 .
$$

Other possible bounds for minimum rank derived from certain easy to compute parameters of the graph were considered, leading to an investigation of the connection between minimum degree of a vertex, $\delta(G)$, and minimum rank [8].

Corollary 2.4 For any graph $G$ and infinite field $F$, $m r^{F}(G) \leq|G|-\delta(G)$.

\section{Main Results}

In here we calculate the minimum rank of graph $G^{j}$. For this purpose we obtained Zero forcing set and the graph parameter $Z(G)$ and the parameter $\xi(G)$ and determined the upper bound and lower bound for maximum nullity $M(G)$. Since we have

$$
\xi(G) \leq M(G) \leq Z(G) .
$$

Then we can achieve minimum rank of graphs (see Table 1).

Theorem 3.1 For all $j \geq 1$ and for all graph such that $Z\left(G^{j}\right)=M\left(G^{j}\right)$, we have $m r\left(G^{j+1}\right) \leq m r\left(G^{j}\right)$.

Proof. It is clear that

$$
m r\left(G^{j}\right)=\left|G^{j}\right|-M\left(G^{j}\right)=|G|-Z\left(G^{j}\right)
$$

and

$$
Z(G) \leq Z\left(G^{2}\right) \leq \cdots \leq Z\left(G^{j}\right),
$$

then

$$
|G|-Z\left(G^{j+1}\right) \leq|G|-Z\left(G^{j}\right) \Rightarrow m r\left(G^{j+1}\right) \leq m r\left(G^{j}\right) .
$$

Proposition 3.2 [1] For each of the following families of graphs, $Z(G)=M(G)$ :

1) Any graph $G$ such that $|G| \leq 6$. (The minimum ranks of all graphs of order at most 7 are available in the spreadsheet [9]).

2) $K_{n}, C_{n}, P_{n}$.

3) Any tree $T$.

4) Some families of special graphs.

By this Proposition we have following Theorem.

Theorem 3.3 For each of the following families of graphs $Z\left(G^{j}\right)=M\left(G^{j}\right)$ :

1) Any graph $G$ such that $|G| \leq 6$.

2) Complete graph and complete multipartite graph.

3) Petersen graph.

4) Wheel graph.

5) Clebsch Graph for $j>1$.

6) Complement of cycle graph $\overline{C_{n}}$, where $n \geq 5$.

Proof. The proof is trivial.

Proposition 3.4 If $P_{n}$ be a path graph, $\left(P_{n}\right)^{n-1}$ is homomorphic to the complete graph $K_{n}$. 
Table 1. Summary of minimum rank results established in this paper.

\begin{tabular}{|c|c|c|c|c|c|}
\hline$G$ & $\left|G^{j}\right|$ & $Z\left(G^{j}\right)$ & $m r\left(G^{j}\right)$ & $j: G^{j} \sim$ Complete Graph & preamble \\
\hline$K_{n}$ & $n$ & $n-1$ & 1 & 1 & \\
\hline$K_{n_{1}, \cdots, n_{p}}$ & $n_{1}+\cdots+n_{p}$ & $n_{1}+\cdots+n_{p}-1$ & 1 & 2 & $j \neq 1$ \\
\hline$W_{n}$ & $n$ & $n-1$ & 1 & 2 & $j \neq 1$ \\
\hline Peterson Graph & 10 & 9 & 1 & 2 & $j \neq 1$ \\
\hline$\overline{C_{n}}$ & $n$ & $n-1$ & 1 & 2 & $j \neq 1$ \\
\hline Clebsch Graph & 16 & 15 & 1 & 2 & $j \neq 1$ \\
\hline$C_{n}$ & $n$ & $2 j$ & $n-2 j$ & {$[n / 2]$} & $j \leq n / 2$ \\
\hline$P_{n}$ & $n$ & $j$ & $n-j$ & $n-1$ & $n>j$ \\
\hline$K_{n} \circ K_{1}$ & $2 n$ & $n$ & $n$ & 3 & $j=2$ \\
\hline$C_{n} \circ K_{1}$ & $2 n$ & $n+2(j-2)$ & $n+2(j-2)$ & {$[n / 2]+2$} & {$[n / 2] \leq j<[n / 2]+2 \quad \& \quad n>5$} \\
\hline$L\left(G^{j}\right)$ & $n$ & - & $n-2$ & - & $j \geq 3 \quad \& \quad n \geq 2$ \\
\hline
\end{tabular}

Theorem 3.5 For all $j<n$, we have $\operatorname{mr}\left(\left(P_{n}\right)^{j}\right)=n-j$.

Proof. (Figure 1) In graph $P_{n}^{j}$, we have $Z\left(P_{n}^{j}\right)=$ $j=M\left(P_{n}^{j}\right)$, because if we start coloring from the end point vertex $u$, this vertex at least is adjacent with $j$ vertices. The vertex $u$ with its $j-1$ adjacent vertices are coloring. The other vertices also are coloring since they are adjacent to coloring vertices, and the number of coloring vertices is $j$, therefore we have $Z\left(P_{n}^{j}\right)=j$. From Corollary 2.2 we have $M\left(P_{n}^{j}\right) \leq Z\left(P_{n}^{j}\right)=j$.

On the other hand with $n-j-1$ contraction of the vertices of $G^{j}$, we reach to the complete graph $K_{j+1}$, and we know that $K_{j+1}$ is a minor for $P_{n}^{j}$. Then we have

$$
j=\xi\left(K_{j+1}\right) \leq \xi\left(P_{n}^{j}\right) \leq M\left(P_{n}^{j}\right) .
$$

Consequently

$$
Z\left(P_{n}^{j}\right)=j=M\left(P_{n}^{j}\right),
$$

thus,

$$
m r\left(P_{n}^{j}\right)=n-j .
$$

(see also [10]).

Proposition 3.6 $\left(C_{n}\right)^{\left[\frac{n}{2}\right]}$ is homomorphic to $K_{n}$.

Theorem $3.7 m r\left(C_{n}^{j}\right)=n-2 j$, for all $j \leq\left[\frac{n}{2}\right]$.

Proof. (Figure 2) In $C_{n}^{j}$ any vertex $u$ is adjacent to $2 j$ vertices, then

$$
M\left(C_{n}^{j}\right) \leq Z\left(C_{n}^{j}\right)=2 j .
$$

On the other hand, $\delta\left(C_{n}^{j}\right)=2 j$. Then

$$
2 j=\delta\left(C_{n}^{j}\right) \leq M\left(C_{n}^{j}\right),
$$

and finally

$$
m r\left(C_{n}^{j}\right)=n-2 j .
$$

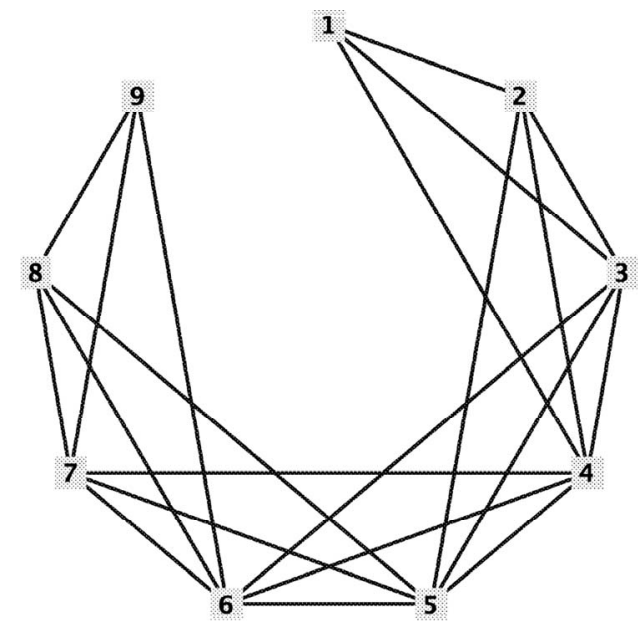

Figure 1. Graph $P_{9}^{3}$.

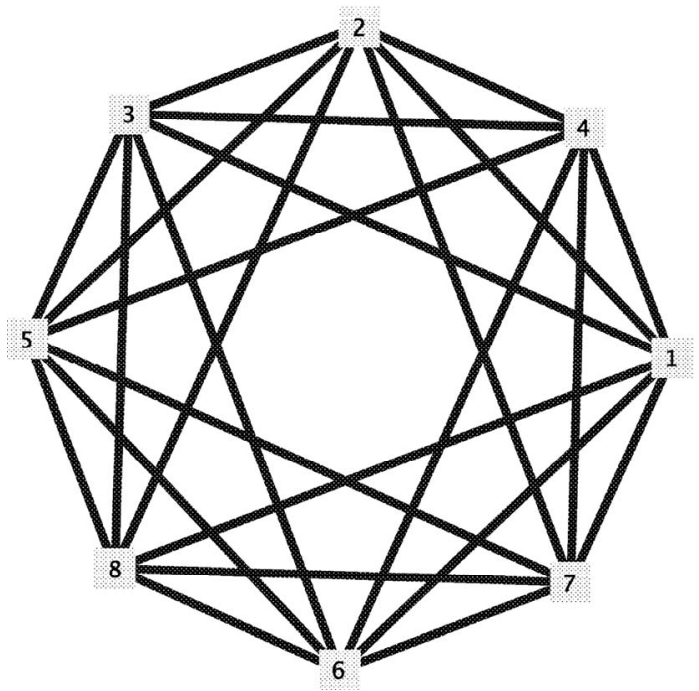

Figure 2. Graph $C_{8}^{3}$. 
Proposition 3.8 For all $j=\left[\frac{n}{2}\right]+2$, we have

$$
\left(C_{n} \circ K_{1}\right)^{j}=K_{2 n} .
$$

Theorem 3.9 For all $j \leq[n / 2]+2$, we have

$$
Z\left[\left(C_{n} \circ K_{1}\right)^{j}\right]=n+2(j-2) .
$$

Proof. (Figures 3, 4, 5) In the $j$ th power of graph $C_{n} \circ K_{1}$ an external vertex $u_{1}$ is adjacent to $3+4(j-2)$ vertices. If we start to coloring of external vertex, then $3+4(j-2)$ vertices are coloring, which $2(j-2)$ colored vertices are in external cycle and the remaining vertices are located in the inner cycle. For more coloring we use the nearest adjacent vertex to $u_{1}$ on the set of external vertices. We call this vertex by $u_{2} .4(j-2)+1$ adjacent vertices to $u_{2}$ are same adjacent vertices to $u_{1}$, and only two of them is different. One of these, which is located on the inner cycle has colored and the another vertex that is located on the external cycle colored from "color-change rule". We continue the process until all vertices are colored on the internal cycle. Finally

$$
Z\left[\left(C_{n} \circ K_{1}\right)^{j}\right]=n+2(j-2) .
$$

Theorem 3.10 For all $[n / 2] \leq j<[n / 2]+2$, and $n \geq 5$ we have $\operatorname{mr}\left(C_{n} \circ K_{1}\right)^{j}=n-2(j-2)$.

Proof. (Figures 3, 4, 5) when we make $[n / 2]$ power of $\left(C_{n} \circ K_{1}\right)$, then its internal cycle reach to complete graph. With contraction of $2 j-1$ vertices to $2 j-3$ vertices of this graph we reach to complete graph of order $n+2(j-2)+1$, then $K_{n+2 j-1}$ is a minor of $\left(C_{n} \circ K_{1}\right)$. On the other hand we have

$$
\begin{aligned}
& n+2(j-2) \\
= & \xi\left(K_{n+2 j-1}\right) \leq \xi\left(\left(C_{n} \circ K_{1}\right)^{j}\right) \leq M\left(\left(C_{n} \circ K_{1}\right)^{j}\right) .
\end{aligned}
$$

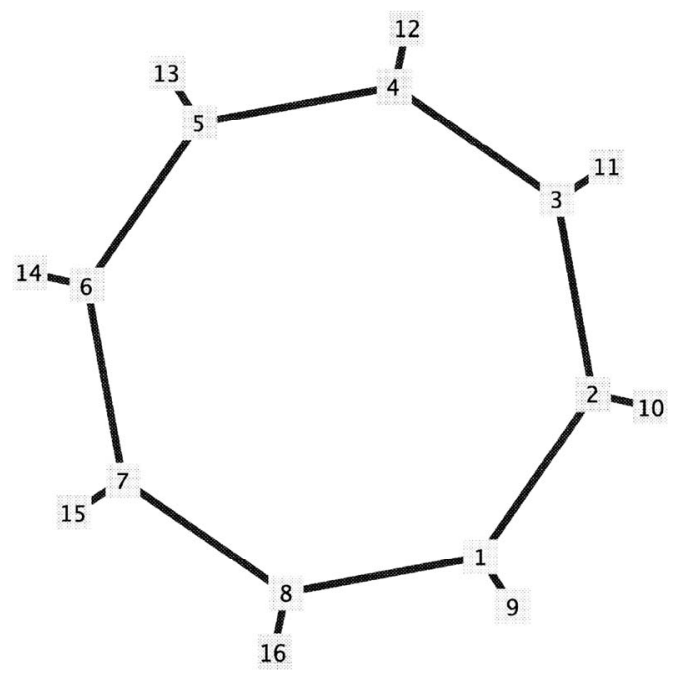

Figure 3. $C_{8} \circ K_{1}$.

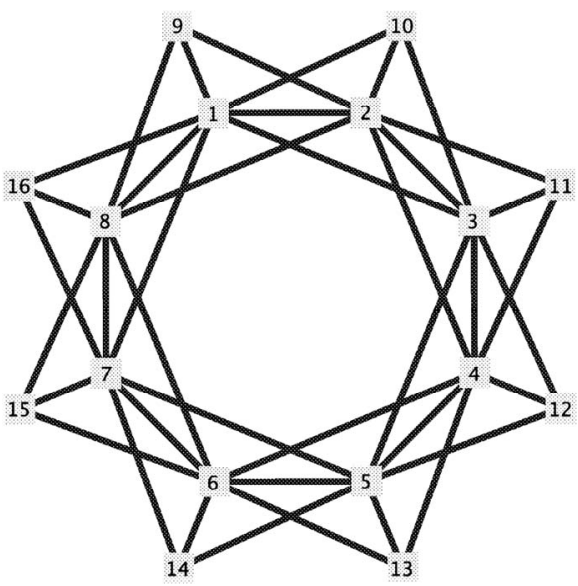

Figure 4. $\left(C_{8} \circ K_{1}\right)^{2}$.

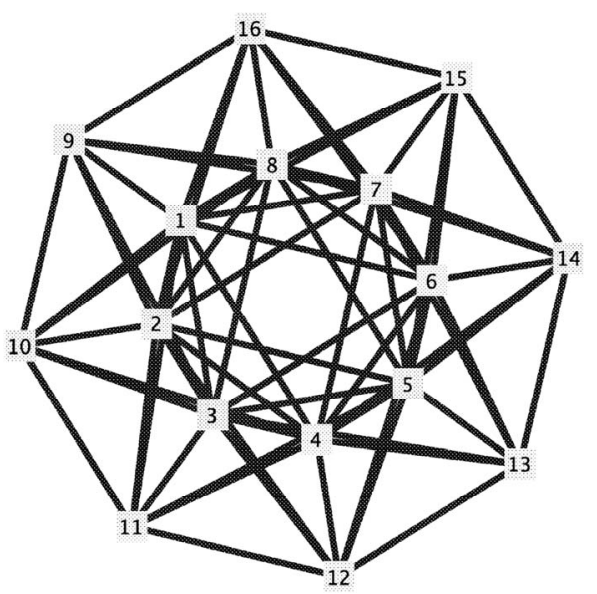

Figure 5. $\left(C_{8} \circ K_{1}\right)^{3}$.

Also according to the previous Theorem, we have $Z\left(\left(C_{n} \circ K_{1}\right)^{j}\right)=n+2(j-2)$, then

$$
M\left(\left(C_{n} \circ K_{1}\right)^{j}\right)=Z\left(\left(C_{n} \circ K_{1}\right)^{j}\right)=n+2(j-2)
$$

and hence $\operatorname{mr}\left(\left(C_{n} \circ K_{1}\right)^{j}\right)=n-2(j-2)$.

Proposition $3.11\left(K_{n} \circ K_{1}\right)^{3}$ is homomorphic to $K_{2 n}$.

Theorem 3.12 $m r\left(\left(K_{n} \circ K_{1}\right)^{2}\right)=n$.

Proof. (Figures 6, 7) With the contraction of $n-1$ external vertices of $\left(K_{n} \circ K_{1}\right)^{2}$ on the vertices which is located in internal cycle, we have the complete graph $K_{n+1}$, then the minor of $\left(K_{n} \circ K_{1}\right)^{2}$, is $K_{n+1}$. So we have

$$
n=\xi\left(K_{n+1}\right) \leq \xi\left(\left(K_{n} \circ K_{1}\right)^{2}\right) \leq M\left(\left(K_{n} \circ K_{1}\right)^{2}\right) .
$$

On the other hand it is trivial that $Z\left(\left(K_{n} \circ K_{1}\right)^{2}\right)=n$. And we have $Z\left(\left(K_{n} \circ K_{1}\right)^{2}\right)=n$ then

$$
m r\left(\left(K_{n} \circ K_{1}\right)^{2}\right)=n \text {. }
$$




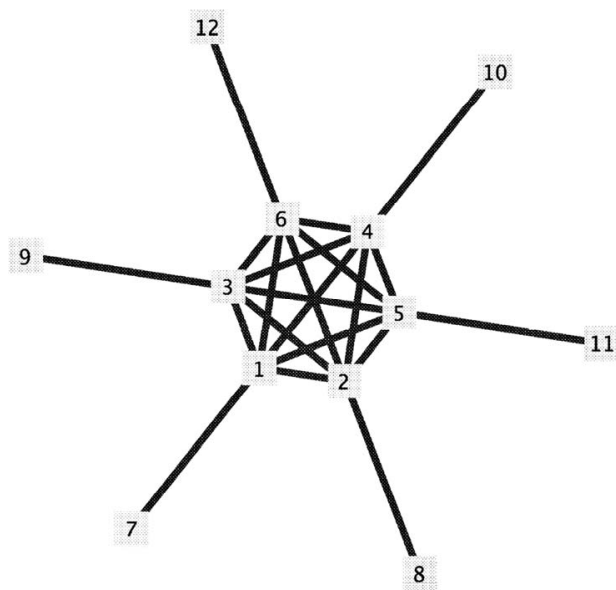

Figure 6. $C_{6} \circ K_{1}$.

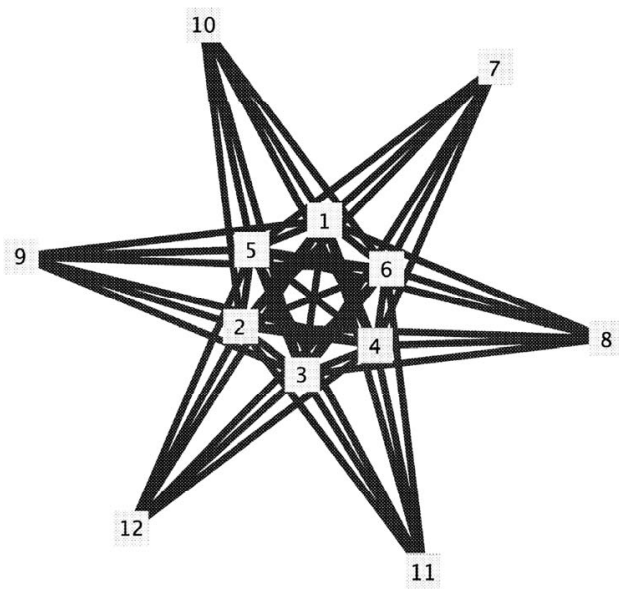

Figure 7. $\left(K_{6} \circ K_{1}\right)^{2}$.

Proposition 3.13 [1] If $G$ has $n \geq 2$ vertices and contains a Hamiltonian path, then $\operatorname{mr}(L(G))=n-2$.

Theorem 3.14 For all $j \geq 3$ and $n \geq 2$ vertices we have, $\operatorname{mr}\left(L\left(G^{j}\right)\right)=n-2$.

Proof. By using the induction on the $|G|$ it can be shown that $G^{3}$ contains a Hamiltonian path. So we have, $\operatorname{mr}\left(L\left(G^{j}\right)\right)=n-2$.

\section{REFERENCES}

[1] AIM Minimum Rank-Special Graphs Work Group, "Zero Forcing Sets and the Minimum Rank of Graphs,” Linear Algebra and Its Applications, Vol. 428, No. 7, 2008, pp. 1628-1648. doi:10.1016/j.laa.2007.10.009

[2] S. Fallat and L. Hogben, "The Minimum Rank of Symmetric Matrices Described by a Graph: A Survey,” Linear Algebra and Its Applications, Vol. 426, No. 2-3, 2007, pp. 558-582. doi:10.1016/j.laa.2007.05.036

[3] L. Hogben, "Spectral Graph Theory and the Inverse Eigenvalue Problem of a Graph," Chamchuri Journal of Mathematics, Vol. 1, No. 1, 2009, pp. 51-72.

[4] F. Barioli, W. Barrett, S. M. Fallat, H. Tracy Hall, L. Hogben, B. Shader, P. van den Dries Che and H. van der Holst, "Zero Forcing Parameters and Minimum Rank Problems,” Linear Algebra and Its Applications, Vol. 433, No. 2, 2010, pp. 401-411. doi:10.1016/j.laa.2010.03.008

[5] L. DeLoss, J. Grout, L. Hogben, T. McKay, J. Smith and G. Tims, "Techniques for Determining the Minimum Rank of a Small Graph,” Linear Algebra and Its Applications, Vol. 432, No. 11, 2010, pp. 2995-3001. doi:10.1016/j.laa.2010.01.008

[6] F. Barioli, S. M. Fallat and L. Hogben, “A Variant on the Graph Parameters of Colin de Verdiere: Implications to the Minimum Rank of Graphs,” Electronic Journal of Linear Algebra, Vol. 13, 2005, pp. 387-404.

[7] L. Hogben and H. van der Holst, "Forbidden Minors for the Class of Graphs $G$ with $\xi(G) \leq 2$," Linear Algebra Applications, Vol. 423, No. 1, 2007, pp. 42-52. doi:10.1016/j.laa.2006.08.003

[8] A. Berman, S. Friedland, L. Hogben, U. G. Rothblum and B. Shader, "An Upper Bound for the Minimum Rank of a Graph,” Linear Algebra and Its Applications, Vol. 429, No. 7, 2008, pp. 1629-1638. doi:10.1016/j.laa.2008.04.038

[9] L. DeLoss, J. Grout, L. Hogben, T. McKay, J. Smith and G. Tims, "Table of Minimum Ranks of Graphs of Order at Most 7 and Selected Optimal Matrices,” http://arxiv.org/abs/0812.0870

[10] American Institute of Mathematics Workshop, "Spectra of Families of Matrices Described by Graphs, Digraphs, and Sign Patterns,” 23-27 October 2006, Palo Alto. 